\title{
Making electrodes by particle stamping for microscopic and electrochemical analysis
}

\author{
Jiyoung Son, Xiao-Ying Yu, Shawn Riechers and Edgar Buck
}

Pacific North West National Laboratory, United States

Studying the electrochemical behavior of nanoparticles using electron microscopy techniques is challenging due to the difficulty in fabricating the working electrode (WE) containing nanoparticles with a consistent loading of particles of interest while minimizing the background signal from the substrate. In this paper, we describe a new nanoparticle stamping method to prepare electrodes contained within a microfluidic platform that is suitable for in-operando electron microscopy [1, 2]. Microscopic and microanalysis techniques, including atomic force microscopy (AFM) and time-of-flight secondary ion mass spectrometry (ToF-SIMS), were used to characterize the as-fabricated electrodes and electrochemical analysis was used to verify electrode performance.

Particle attachment is not a new concept in electrochemistry and many powdered materials have been deposited directly on an electrode surface consisting of polymer composites, graphite, or silver [3, 4]. The applied materials were then covered with either a layer of thick-film or clay. These studies demonstrated both microscopy/spectroscopy and electrochemistry of the nanoparticle electrode systems. However, the electrodes exhibited poor reproducibility owing to the low conductivity of polymer composites, zeolites, and clays. Additionally, thick-film deposits can generate high currents which will interfere with the characteristics of target particle materials. Thus, controlling the amount of the material dispensed onto a surface is critical in the process of direct deposition on an electrode (DDE) [5, 6].

The electrodes with attached particles were fabricated as a part of the established microfluidic device for multimodal spectroscopy and microscopy of liquids, $[1,2]$ which is called the System for Analysis at the Liquid-Vacuum Interface Electrochemical cell (SALVI E-cell). Silver conductive epoxy (CW2400, Chemtronics) was used as the substrate material for particle deposition onto the WE surface by sequence stamping. Briefly, a thin layer of silver conductive epoxy was squeezed onto a plate. A custom-made stamping tool with a stamping surface of $1.5 \times 1.5 \mathrm{~mm}^{2}$ (polymethyl methacrylate, PMMA) was used to pick up a layer of $\sim 200 \mu \mathrm{m}$ thick of the silver epoxy. Then epoxy layer was used to pick up particles from the dish. Finally, the stamped particles were pressed and released to the surface of a silicon nitride (SiN) membrane supported on a silicon frame (Norcada, $0.5 \times 0.5 \mathrm{~mm}$ on a $200 \mu \mathrm{m} \mathrm{Si}$ frame) (Figure 1C). Approximately $20 \mathrm{mg}$ cerium oxide nanoparticles $\left(\mathrm{CeO}_{2}\right.$, US research materials $\mathrm{Inc}, 10 \mathrm{~nm}$ mean diameter) were used in each device.

Electrochemical analysis (Electrochemical station, $\mathrm{CH}$ instruments; $660 \mathrm{~h}$ ) of the nanoparticle electrodes was performed with a scan rate of $100 \mathrm{mV} / \mathrm{s}$. A $0.1 \mathrm{mM}$ potassium chloride $(\mathrm{KCl})$ solution was used as the electrolyte. Cyclic voltammetry (CV) scans (Figure 1C) of the fabricated device using the sequence stamping approach show the unique electrochemical profiles of $\mathrm{CeO}_{2}$ nanoparticles. This result was different from that of the conductive epoxy control CV shown in Figure 1D. For example, there were unique $\mathrm{CV}$ peak profiles at $-0.1 \mathrm{~V}$ (from $1 \mathrm{~V}$ to $-1 \mathrm{~V}$ scan) and $0.7 \mathrm{~V}$ (from $-1 \mathrm{~V}$ to $1 \mathrm{~V}$ scan), showing different features from the control CV profile. Moreover, we characterized the stamped electrode surfaces using AFM and ToF-SIMS via 2D imaging shown in Figure 2. AFM images (Figure 2A-D) show the morphology of nanoparticle aggregation on the stamped surface ranging from 40 to $130 \mathrm{~nm}$ in height and the ToF-SIMS 2D image (Figure $2 \mathrm{E}$ ) verifies the presence of $\mathrm{CeO}_{2}$ particles on the stamped surface. Both microanalysis and imaging techniques provided valuable insight for optimization and assisted method development. Nanomaterials can be analyzed relatively conveniently for their electrochemical properties using this new particle attachment method in a microfluidic cell. We provide a good tool for rapid nanomaterial electrochemical analysis. More importantly, this approach permits in situ SEM analysis and 
imaging and allows nanoparticle electrochemical performance evaluation using multi-dimensional microanalysis.
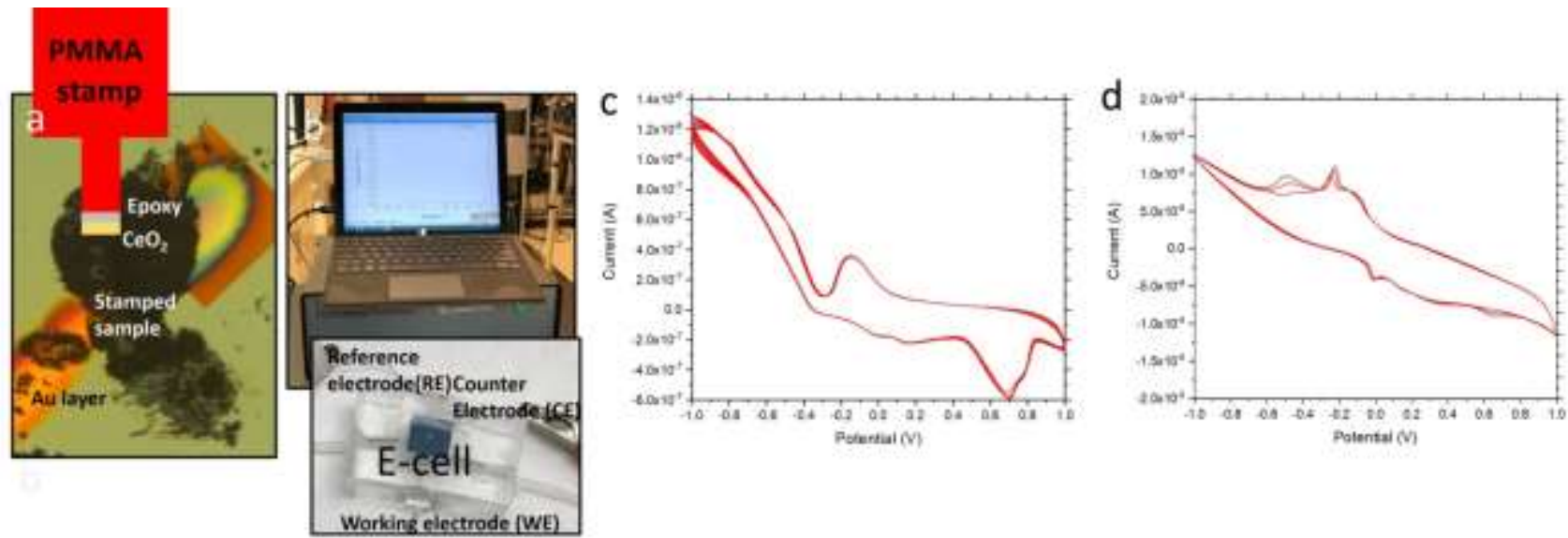

Figure 1. The electrochemical analysis setup, (a) a photo showing the $\mathrm{CeO} 2$ particles stamped onto the gold conductive layer using the PMMA stamp (b) the electrochemical station with the data computer, the fabricated SALVI E-cell, (c) Cyclic voltammograms of the sequence stamped $\mathrm{CeO} 2$ device at a scan rate of $100 \mathrm{mV} / \mathrm{s}$, (d) and the conductive epoxy applied control device.
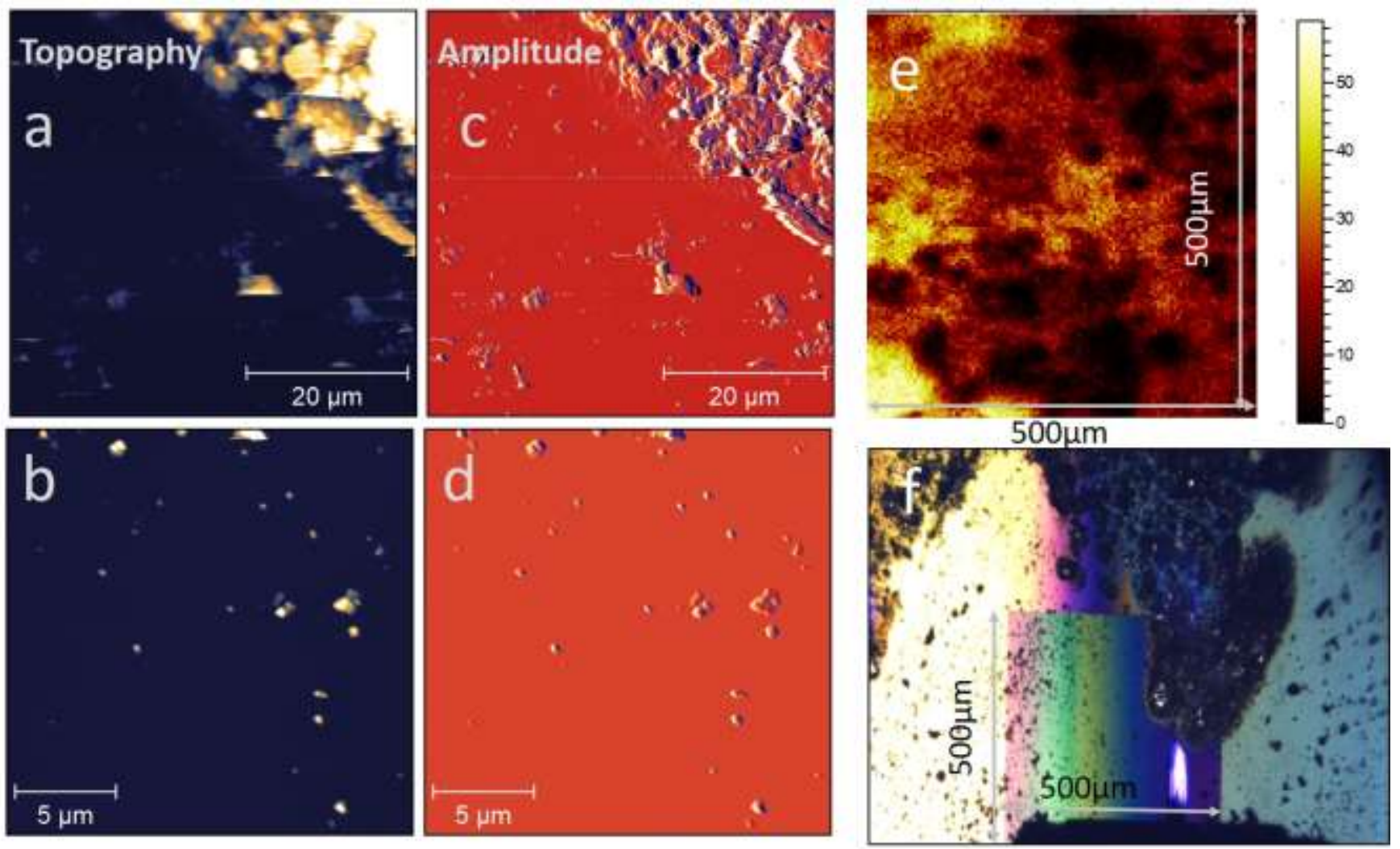

Figure 2. AFM, ToF-SIMS $2 \mathrm{D}$ and optical images of $\mathrm{CeO} 2$ and stamped on the SiN substrate: (a) AFM topography images of the stamp surface and (b) the surface with $\mathrm{CeO} 2$ deposited, (c) AFM amplitude of the stamp surface, (d) $\mathrm{CeO} 2$ deposited surface, (e) a ToF-SIMS 2D image of the stamped surface with $\mathrm{CeO} 2$ particles showing Ce m/z+ 139.90, and (f) an optical image of the sequence stamped SiN window surface and AFM probe at the imaging location shown in a-d. 


\section{References}

[1] Yang et. Al, Lab Chip, 2011. 11(15): p. 2481-4

[2] Yang et. Al, Journal of Vacuum Science \& Tech A: Vacuum, Surfaces, and Films, 2011. 29(6)

[3] Doménech-Carbó et. Al, Fresenius J Anal Chem 2001. 369: p. 6.

[4] Marken et. Al. Collection of Czechoslovak Chem Comm, 2002, 67(2), 163-208

[5] Ghosh et. Al. Journal of ACS, 1983, 105, p3.

[6] Son et. Al. Micromachines 2021, 12(1), 60. 